\title{
Inhaled Corticosteroid-Containing Treatment Escalation and Outcomes for Patients with Asthma in a U.S. Health Care Organization
}

\author{
Lindsay G. S. Bengtson, PhD; Yanni Yu, DSc; Weijia Wang, MSc; Feng Cao, PhD; Erin M. Hulbert, MS; \\ Ryan Wolbeck, MS; Caitlin A. Elliott, MS; and Ami R. Buikema, MPH
}

\begin{abstract}
BACKGROUND: Asthma is a common disorder that affects approximately $8 \%$ of the U.S. population. Treatment guidelines indicate inhaled corticosteroids (ICS) as the mainstay treatment, yet poor asthma control is common among ICS-treated patients. Treatment escalation (ICS dose increase and other controller therapy add-ons) is used to manage symptoms. Real-world studies of postescalation outcomes may inform treatment decisions.
\end{abstract}

OBJECTIVES: To (a) describe characteristics and treatment patterns among asthma patients who escalated treatment and (b) assess outcomes (exacerbations, uncontrolled asthma, and health care resource utilization [HCRU]) after escalation.

METHODS: The study cohort was identified from a large U.S. administrative claims database via ICD-9-CM codes for asthma (493.xx on $\geq 2$ dates) and initiation (defining index date) of long-term (>1 fill) ICS-containing treatment between January 1, 2009, and September 30, 2014. One year of continuous enrollment was required before and after the index date. Escalation was defined as $\geq 1$ of the following: ICS dose increase; a switch between ICS, long-acting beta-2 agonists (LABA), or leukotriene modifiers (LTRM) to a different ICS, LABA, or LTRM; or add-on of controller medications (e.g., antibody biologic). Escalation patterns were examined. Rates of exacerbation (defined by inpatient admission, emergency department [ED] visit, or office visit with a pharmacy claim for an oral corticosteroid [0CS] within 7 days) and occurrence of uncontrolled asthma (defined by $>4$ fills for a short-acting beta agonist [SABA] in a 1-year period, $\geq 10 \mathrm{CS}$ fill, or $\geq 1$ asthma-related ED visit or inpatient admission) were calculated. Perpatient-per-year (PPPY) HCRU was estimated.

RESULTS: Among 35,126 patients (mean [SD] age 38 [16] years) who initiated long-term ICS-containing treatment, 5,044 (14\%) patients escalated their index regimens at 136 (105) days post-index (i.e., pre-escalation period). The most frequent changes, alone or in combination, included ICS dose increase $(68 \%)$ or LABA $(27 \%)$ or LTRM $(25 \%)$ add-ons. Before escalation, the exacerbation rate was 1.60 (5.10) PPPY, and 1,108 (22\%) patients experienced exacerbation. During the postescalation period of $251.6(138.9)$ days, the exacerbation rate was 0.75 (2.9) PPPY, and 1,038 (21\%) patients experienced exacerbation. A majority $(>85 \%)$ of exacerbations in the periods before and after escalation were associated with an office visit plus an OCS pharmacy claim within 7 days. Uncontrolled asthma was experienced by $41.5 \%$ and $41.0 \%$ of patients before and after escalation, respectively. Ambulatory care visits were common before (mean [SD] 24.0 [26.7] allcause and 8.5 [13.4] asthma-related PPPY) and after escalation (19.3 [21.3] all-cause and 4.6 [8.1] asthma-related PPPY).

CONCLUSIONS: Among asthma patients who initiated a long-term ICScontaining regimen, approximately $14 \%$ escalated therapy within a year of initiation. Yet, $21 \%$ of those patients had $\geq 1$ exacerbation, and $41 \%$ of patients had uncontrolled asthma within 1 year after treatment escalation. The results demonstrate an unmet need among asthma patients who escalated their ICS-containing treatment.

J Manag Care Spec Pharm. 2017;23(11):1149-59

Copyright $\odot 2017$, Academy of Managed Care Pharmacy. All rights reserved.

\section{What is already known about this subject}

Asthma is a common disorder that affects approximately $8 \%$ of the U.S. population, with inhaled corticosteroids (ICS) as the mainstay treatment, yet many ICS-treated patients have uncontrolled symptoms.

ICS dose escalation is the first approach for inadequately controlled asthma, with ICS dose escalation and/or add-on therapy with long-acting beta-2 agonists (LABA) or leukotriene modifiers (LTRM) commonly used for more severe or uncontrolled asthma. It has been reported that more than $50 \%$ of patients who experience dose escalation continue to have frequent exacerbations.

\section{What this study adds}

Among patients who initiated long-term ICS-containing treatment for asthma, 14\% escalated their regimens within 1 year of treatment initiation, and the most common modifications, alone or in combination, included ICS dose increase or LABA or LTRM add-ons. During the postescalation period (up to 1 year), $21 \%$ of patients had exacerbations, and $41 \%$ of patients had uncontrolled asthma. Despite treatment escalation, fills for rescue medications (shortacting beta agonists and oral corticosteroids) remained high in the postescalation period relative to the pre-escalation period.

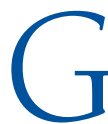
lobally, more than 334 million individuals may be affected by asthma. ${ }^{1}$ In the United States, the Centers for Disease Control and Prevention estimated that the prevalence of asthma was $7.8 \%$ in $2015 .^{2}$ Asthma patients in the United States experience approximately 10.5 million asthma-related office visits, 1.3 million outpatient hospital visits, and 1.6 million emergency department (ED) visits per year, ${ }^{3}$ resulting in substantial financial burden-an estimated $\$ 56$ billion annually. ${ }^{4}$

Treatment guidelines for asthma control from the National Heart Lung and Blood Institute (NHLBI) and Global Initiative for Asthma (GINA) advise a stepwise approach, since the frequency and severity of symptoms dictate the course of treatment. ${ }^{5,6}$ Inhaled corticosteroids (ICS) represent the mainstay of asthma treatment, with an increase of the ICS dose being the first response to inadequate control. ${ }^{5,7,8}$ When asthma is inadequately controlled, treatment may be escalated with increased ICS dose and/or add-on therapy with long-acting 


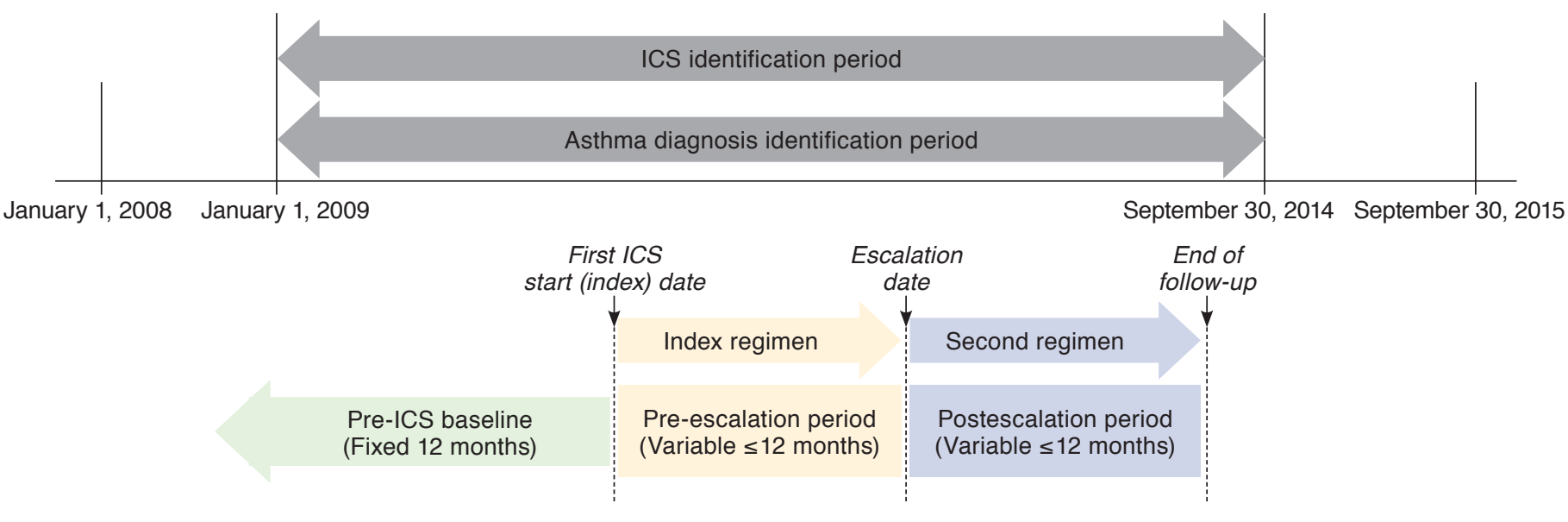

ICS = inhaled corticosteroid .

beta-2 agonists (LABA), leukotriene modifiers (LTRM), antibody biologics, or oral corticosteroids (OCS).,6

Despite advances in available treatments, $\geq 40 \%$ of patients exhibit asthma that is not well controlled, which may require escalated treatment. ${ }^{9}$ Even with escalated pharmacotherapy, $>50 \%$ of patients may experience frequent exacerbations-episodes of worsening coughing, wheezing, shortness of breath, and/or chest tightness_-suggesting a need for better strategies to improve asthma control. ${ }^{10}$ Exacerbations are associated with nearly double the health care costs relative to patients without exacerbations ( $\$ 9,223$ vs. $\$ 5,011$, respectively). ${ }^{11}$ Studies have shown a continuing burden of uncontrolled and/or severe asthma accompanied by exacerbations, which demonstrates an unmet need in current asthma care. ${ }^{12-15}$ Estimates of the financial effect of this burden include 2.9-fold higher health care costs among patients with severe asthma (characterized by exacerbations) versus persistent asthma (without exacerbations) in an administrative claims-based study. ${ }^{13}$ Understanding treatment patterns and outcomes among patients with asthma who initiate a long-term ICS-containing regimen in a realworld setting can further inform asthma management. Several administrative claims studies have examined treatment patterns alone or assessed outcomes related to different guidelinerecommended treatment intensities. ${ }^{13,16,17}$ However, treatment escalation and outcome definitions vary among studies, and it is unclear to what degree exacerbations and/or uncontrolled asthma persist after escalation; moreover, health care utilization and costs among patients who have escalated treatment are not well understood.
The objectives of this study were to (a) describe characteristics and treatment patterns among asthma patients who escalated their index ICS-containing treatments within 1 year of initiation and (b) assess asthma exacerbations and health care utilization after escalation.

\section{Methods}

\section{Sample Selection}

This study was performed using the Optum Research Database, a large U.S. health care claims database representing approximately 12.6 million commercial plan members annually. This study used medical and pharmacy claims with linked enrollment information from January 1, 2008, to September 30, 2015.

The study population included commercial health plan enrollees with evidence of asthma who initiated a long-term (> 1 fill) ICS or ICS-containing regimen between January 1 , 2009, and September 30, 2014 (Figure 1); the initial ICS fill date was the index date. Patients who had an ICS dose increase, switched from an ICS, LABA, or LTRM to a different ICS, LABA, or LTRM or added on controller medications, including LABA, LTRM, mast cell stabilizer, methylxanthine, and omalizumab, within the first year post-index were assigned to the escalation group, which was the study cohort of interest. Those patients whose regimen was unchanged were assigned to the unchanged group, which was included for baseline characteristics only. Those who had a step down in therapy, defined as an ICS dose decrease, discontinuation of 1 or more controller medications, or both, were assigned to a step-down group, which was not included in this analysis.

Inclusion and Exclusion Criteria. Inclusion criteria were based on ICS therapy and asthma diagnosis. ICS use was 
evidenced by a prescription fill for an ICS or ICS-containing therapy (identified by National Drug Code [NDC] numbers), from January 1, 2009, to September 30, 2014. All asthma controller medications filled on or within 7 days after the index date were defined as part of the first regimen.

Asthma was identified by $\geq 2$ medical claims on 2 different dates with an asthma diagnosis code (using International Classification of Diseases, Ninth Revision, Clinical Modification [ICD-9-CM] code 493.xx) in any position between January 1, 2009, and September 30, 2014. At least 1 of the claims must have occurred before or on the index date. In addition, patients had to be aged $\geq 12$ years as of the index date and had to have continuous enrollment in their health plans with medical and pharmacy benefits for 12 months before and $\geq 12$ months after the index date.

Inclusion in the escalation group required evidence of an ICS or ICS-containing therapy dose increase; a switch among ICS, LABA, or LTRM; or an add-on of another controller within 12 months after the index date, with the date of the first of these changes defined as the escalation date. Patients in the escalation group had to have $\geq 12$ months of continuous enrollment after the escalation date. Patients with $\geq 1$ additional fill indicating continuation of the index treatment regimen within 12 months after the index date were included in the unchanged group.

Patients were excluded if they had ICS, LABA, or LTRM use or a diagnosis code for acute respiratory failure (ICD-9-CM code 518.81) during the 12 months before the index date or if they did not fill another prescription for an ICS-containing regimen within 1 year after the index date. Patients with any of the following during the entire study period were excluded: (a) $\geq 1$ medical claim with diagnoses for chronic obstructive pulmonary disease, cystic fibrosis, or interstitial lung disease, in any position or (b) evidence of ipratropium or a long-acting muscarinic antagonist (LAMA) and/or LAMA/LABA use following an asthma diagnosis (these medications were not approved for asthma during the study period). In addition, patients in the step-down group were excluded.

\section{Study Design}

The study design included 3 analytic periods (Figure 1). For the escalation and unchanged groups, demographic and clinical characteristics were described for the pre-ICS baseline period - the 1-year period before the index date. For the escalation group only, treatment patterns, health care resource utilization (HCRU) and costs, and exacerbations were described for the pre-escalation period, a variable period of $\leq 12$ months from the index date until the date at which the treatment regimen was escalated, and for the postescalation period, a variable period of $\leq 12$ months from the escalation date to the date of either a second escalation (if applicable) or the end of the 12-month post-index follow-up period.

\section{Measures}

Patient Characteristics. Demographic characteristics measured for the escalation and unchanged groups included age at index, gender, and geographic region, ${ }^{18}$ determined in the pre-ICS baseline period, and length (in days) of the followup periods. Clinical characteristics included a continuous and categorized $(0,1-2,3-4,5+)$ Charlson comorbidity score, calculated based on the presence of diagnosis codes on medical claims during the pre-ICS baseline period. ${ }^{19}$ The 5 most prevalent comorbidity categories based on the Agency for Healthcare Research and Quality (AHRQ) categorization also were recorded. In addition, lower respiratory tract infection was noted based on the presence of ICD-9-CM codes (Appendix A, available in online article).

Treatment Patterns. Treatment patterns were measured for the escalation group during the pre- and postescalation periods. Data were collected on index regimen medications (determined based on NDC numbers for ICS, LABA, LTRM, methylxanthine, and mast cell stabilizer and on NDC numbers or Healthcare Common Procedure Coding System codes for omalizumab), in addition to medications that were added or switched in the escalation regimen. Treatment patterns of asthma rescue medications (short-acting beta agonist [SABA] and OCS) also were assessed.

The mean ICS daily dose was computed for each ICS or ICS/ LABA pharmacy claim. For each NDC number, the prescribing information was used to determine the number of units (i.e., the number of vials or canisters) and the micrograms in a unit. The total dose was calculated as the number of units multiplied by the micrograms per unit. The mean daily dose was calculated as the total dose divided by the days supply recorded on the pharmacy claim and was categorized as low, medium, or high, according to the NHLBI guidelines. ${ }^{5}$

\section{Outcomes}

Exacerbations. An asthma exacerbation was identified by any of the following conditions: hospitalization with asthma ICD9-CM diagnosis code 493.xx in the primary position; ED or urgent care facility visit with asthma ICD-9-CM diagnosis code 493.xx in the primary position; and office visit with asthma ICD-9-CM code 493.xx in any position and a pharmacy claim for a systemic (oral or injectable) corticosteroid within \pm 7 days of the claim for this office/outpatient visit.

If 2 or more exacerbation events occurred within 15 days of each other, they were considered a single exacerbation episode. ${ }^{20}$ The type of exacerbation episode was defined based on care setting: inpatient, ED, or office visit. 


\section{FIGURE 2 Attrition and Sample Selection}

Commerical enrollees with evidence of asthma on $\geq 2$ medical claims (ICD-9-CM diagnosis of 493.xx) on unique dates January 1, 2009-September 30, 2014 and $\geq 1$ pharmacy claim for an ICS or ICS-containing regimen (on date defining index) after first medical claim for asthma $\mathrm{N}=534,929(100 \%)$
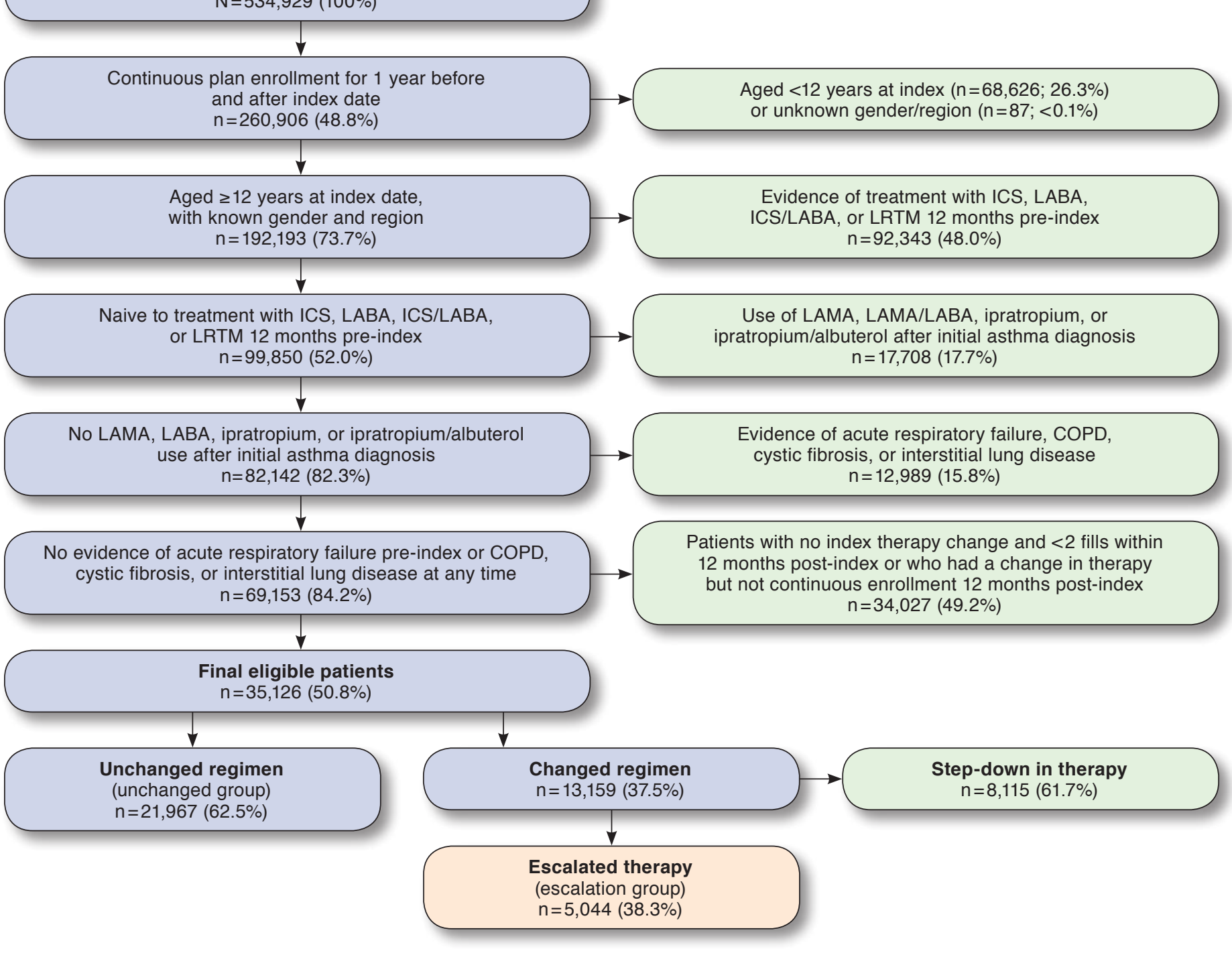

COPD = chronic obstructive pulmonary disease; ICS = inhaled corticosteroid; ICD-9-CM=International Classification of Diseases, Ninth Revision, Clinical Modification; $L A B A=$ long-acting beta-2 agonist; LAMA = long-acting muscarinic antagonist; LRTM =leukotriene modifier.

A proxy for uncontrolled asthma was derived from previous research ${ }^{21}$; asthma was considered uncontrolled if any of the following conditions were met:

- $\geq 4$ pharmacy claims for a SABA per year or the equivalent number of SABA claims based on a shorter follow-up period (i.e., $\geq 2$ pharmacy claims for a SABA in 180 days)
No continuous enrollment $n=274,023(51.2 \%)$
Aged $<12$ years at index $(n=68,626 ; 26.3 \%)$

or unknown gender/region $(n=87 ;<0.1 \%)$

Evidence of treatment with ICS, LABA $\mathrm{n}=92,343(48.0 \%)$
Evidence of acute respiratory failure, COPD, is, or interstitial lung disease $=12,989(15.8 \%)$
Patients with no index therapy change and $<2$ fills within 12 months post-index or who had a change in therapy $n=34,027(49.2 \%)$
- $\geq 1$ prescription claim for an OCS

- $\geq 1$ medical claim for asthma-related ED or hospitalization with ICD-9-CM diagnosis code 493.xx in any position

Health Care Resource Utilization. HCRU was calculated as all-cause (claims containing any diagnoses codes) and asthma-related (only claims containing an asthma diagnosis 


\begin{tabular}{|c|c|c|c|}
\hline & $\begin{array}{l}\text { Escalation Group } \\
\qquad(\mathrm{n}=5,044)\end{array}$ & $\begin{array}{l}\text { Unchanged Group } \\
\qquad(\mathrm{n}=21,967)\end{array}$ & $P$ Value \\
\hline \multicolumn{4}{|l|}{ Baseline demographic characteristics } \\
\hline Age, years, mean (SD) & $(16.4)$ & $(16.2)$ & $<0.001$ \\
\hline Female, $\mathrm{n}(\%)$ & $(60.6)$ & 12,692 & 0.001 \\
\hline \multicolumn{4}{|l|}{ Geographic region, n (\%) } \\
\hline Northeast & $(13.7)$ & $(12.2)$ & 0.001 \\
\hline Midwest & $(25.8)$ & $(27.8)$ & $<0.001$ \\
\hline South & $(40.5)$ & $(39.7)$ & 0.017 \\
\hline West & $(20.0)$ & $(20.2)$ & 0.166 \\
\hline \multicolumn{4}{|l|}{ Baseline clinical characteristics } \\
\hline Charlson comorbidity score, mean (SD) & $(0.8)$ & $(0.8)$ & 0.008 \\
\hline \multicolumn{4}{|l|}{ Charlson comorbidity score category, n (\%) } \\
\hline 0 & $(26.6)$ & $(24.3)$ & $<0.001$ \\
\hline $1-2$ & $(69.0)$ & 15,615 & $<0.001$ \\
\hline $3-4$ & (3.9) & $(4.0)$ & 0.898 \\
\hline $5+$ & $(0.6)$ & $(0.6)$ & 0.737 \\
\hline \multicolumn{4}{|l|}{ Top 5 AHRQ comorbidities, n (\%) } \\
\hline Respiratory infections & $(56.3)$ & 10,987 & $<0.001$ \\
\hline Other upper respiratory disease & $(42.1)$ & 8,600 & $<0.001$ \\
\hline Other lower respiratory disease & $(41.3)$ & $(35.8)$ & $<0.001$ \\
\hline Immunizations and screening for infectious disease & $(38.0)$ & $(35.0)$ & 0.005 \\
\hline Other connective tissue disease & $(27.3)$ & $(25.3)$ & 0.019 \\
\hline Lower respiratory tract infection, n (\%) & $(24.0)$ & $(21.2)$ & $<0.001$ \\
\hline
\end{tabular}

code in any position). Costs were calculated as supplemental information with related methods, and results are described in Appendix B (available in online article).

HCRU was assessed for ambulatory visits (office and outpatient), ED visits, and inpatient admissions for the pre- and postescalation periods. For each utilization category, the presence of at least 1 utilization and the number of visits during the period (annualized as per patient per year [PPPY]) were captured. The count of ambulatory visits was enumerated as 1 visit per provider per day.

\section{Analyses}

The primary data analysis was descriptive in nature. Counts and percentages were reported for categorical variables; means and standard deviations (SDs) were reported for continuous variables. Descriptive analyses that accounted for a variable length of observation time (e.g., PPPY) were performed where appropriate.

Statistical comparisons between the escalation group and unchanged group were performed for the pre-ICS baseline period to describe differences in demographic and clinical characteristics. T-tests were used to compare means of continuous variables between groups; chi-square tests were used for comparison of categorical variables. If presented with low cell counts, Fisher's exact testing was used. $P$ values $<0.05$ were considered statistically significant. Paired t-tests were used to compare the means of continuous variables between the pre- and postescalation periods; comparison of categorical/ binary variables between periods was made using McNemar's test. If presented with low cell counts, McNemar's exact test was used. All statistical analyses were conducted with SAS version 9.4 (SAS Institute, Cary, NC).

\section{Results}

\section{Study Population}

Initially, 534,929 patients were eligible for inclusion in the study based on evidence of asthma and pharmacy claims for an ICS or ICS-containing regimen (Figure 2). After applying additional inclusion and exclusion criteria, a final cohort of 35,126 patients remained. There were 5,044 patients who escalated their index therapy within 12 months (escalation group); 21,967 patients who had at least 2 fills of their index medication and did not change their index therapy within 12 months (unchanged group); and 8,115 patients who stepped down their index therapy and were not included in subsequent analyses.

The mean (SD) age of patients was lower in the escalation group (37.5 [16.4] years) than the unchanged group (39.0 [16.2] years, $P<0.001$; Table 1). There were more females $(60.6 \%)$ in the escalation group than in the unchanged group $(57.8 \%$, $P=0.001$ ). The escalation group had a lower mean Charlson 


\section{TABLE 2 Treatment for the Escalation Group (Before and After Escalation) and the Unchanged Group}

\begin{tabular}{|c|c|c|c|c|c|c|c|c|}
\hline \multirow{3}{*}{$\begin{array}{l}\text { Medication Class }^{\mathbf{a}} \\
\text { Period length, days, mean (SD) }\end{array}$} & \multicolumn{4}{|c|}{$\begin{array}{l}\text { Escalation Group } \\
\qquad(\mathrm{n}=5,044)\end{array}$} & \multicolumn{2}{|c|}{$\begin{array}{l}\text { Unchanged Group } \\
\qquad(\mathrm{n}=21,967)\end{array}$} & \multicolumn{2}{|c|}{$P$ Values } \\
\hline & \multicolumn{2}{|c|}{ Before Escalation } & \multicolumn{2}{|c|}{ After Escalation } & \multicolumn{2}{|c|}{ Index Regimen } & \multirow{2}{*}{$\begin{array}{c}\text { Escalation Group } \\
\text { (Before vs. After } \\
\text { Escalation) } \\
<0.001\end{array}$} & \multirow{2}{*}{ 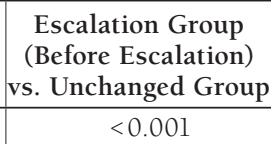 } \\
\hline & 136.1 & $(105.2)$ & 251.6 & (138.9) & 365.0 & (1.4) & & \\
\hline Period length, days, median (p25, p75) & 110 & $(41,218)$ & 365 & $(101,365)$ & 365 & $65,365)$ & & \\
\hline \multicolumn{9}{|l|}{ ICS dose, ${ }^{\mathrm{b}} \mathrm{n}(\%)$} \\
\hline Low dose & 1,520 & $(30.1)$ & 240 & $(4.8)$ & 3,800 & $(17.3)$ & $<0.001$ & $<0.001$ \\
\hline Medium dose & 1,475 & $(29.2)$ & 1,207 & $(23.9)$ & 5,209 & $(23.7)$ & $<0.001$ & $<0.001$ \\
\hline High dose & 215 & $(4.3)$ & 830 & $(16.5)$ & 1,012 & $(4.6)$ & $<0.001$ & 0.289 \\
\hline \multicolumn{9}{|l|}{ ICS/LABA dose, ${ }^{\mathrm{b}}$ n (\%) } \\
\hline Low dose & 670 & $(13.3)$ & 222 & $(4.4)$ & 2,583 & (11.8) & $<0.001$ & 0.003 \\
\hline Medium dose & 793 & $(15.7)$ & 1,095 & $(21.7)$ & 4,764 & $(21.7)$ & $<0.001$ & $<0.001$ \\
\hline High dose & 392 & $(7.8)$ & 1,720 & $(34.1)$ & 4,671 & $(21.3)$ & $<0.001$ & $<0.001$ \\
\hline LABA, n (\%) & 12 & $(0.2)$ & 32 & $(0.6)$ & 18 & $(0.1)$ & 0.001 & 0.003 \\
\hline Leukotriene modifier, n (\%) & 188 & $(3.7)$ & 1,393 & $(27.6)$ & 409 & $(1.9)$ & $<0.001$ & $<0.001$ \\
\hline \multicolumn{9}{|l|}{ Other controller medications, $\mathrm{n}(\%)$} \\
\hline Mast cell stabilizer & 1 & $(0.0)$ & 2 & $(0.0)$ & 0 & $(0.0)$ & 0.021 & 0.187 \\
\hline Methylxanthine & 1 & $(0.0)$ & 22 & $(0.4)$ & 3 & $(0.0)$ & $<0.001$ & 0.563 \\
\hline Omalizumab & 2 & $(0.0)$ & 21 & $(0.4)$ & 0 & $(0.0)$ & $<0.001$ & 0.035 \\
\hline \multicolumn{9}{|l|}{ Rescue medications, n (\%) } \\
\hline SABA & 2,981 & $(59.1)$ & 2,832 & $(56.2)$ & 15,834 & $(72.1)$ & 0.001 & $<0.001$ \\
\hline OCS & 1,501 & $(29.8)$ & 1,548 & $(30.7)$ & 7,002 & $(31.9)$ & 0.267 & 0.004 \\
\hline OCS (chronic use)c & 6 & $(0.1)$ & 7 & $(0.1)$ & 52 & $(0.2)$ & 0.049 & 0.103 \\
\hline \multicolumn{9}{|c|}{$\begin{array}{l}\text { a Medication classes are not mutually exclusive; patients taking medications from multiple classes are included in each category. } \\
\text { bClassified according to NHLBI. } \\
\text { cDefined as } \geq 90 \text { consecutive days of OCS coverage. } \\
\text { ICS = inhaled corticosteroid; LABA = long-acting beta-2 agonist; NHLBI=National Heart, Lung, and Blood Institute; OCS = oral cortacosteroid; SABA= short-acting } \\
\text { beta-2 agonist; SD = standard deviation. }\end{array}$} \\
\hline
\end{tabular}

comorbidity score than the unchanged group (0.87 [0.76] vs. $0.90[0.77], P=0.008$ ) but a greater percentage of patients with lower respiratory tract infections (escalation group: $24.0 \%$ vs. unchanged group: $21.2 \%, P<0.001$ ). Several of the top 5 most prevalent AHRQ comorbidities were more common among the escalated group than the unchanged group, including any respiratory infections (escalation group: $56.3 \%$ vs. unchanged group: $50.0 \%, P>0.001$ ) and other upper respiratory disease (escalation group: $42.1 \%$ vs. unchanged group: $39.2 \%, P<0.001$ ).

\section{Treatment Patterns}

Treatment patterns were examined in the escalation and unchanged groups during the post-index period, as shown in Table 2. The mean (SD) duration of the pre-escalation period was 136.1 (105.2) days with an observation period of 365.0 (1.4) days among unchanged patients. For patients in the escalation group, the mean (SD) length of treatment for the escalated regimen in the postescalation period was 251.6 (138.9) days. Over 30\% of patients who escalated were treated with low-dose ICS before escalation (vs. 17.3\% among unchanged, $P<0.001$ ), whereas $4.8 \%$ were treated with lowdose ICS after escalation (all pre- vs. postescalation ICS dose comparisons $P<0.001)$. The most frequent dosage for combined ICS/LABA was the medium dose (15.7\%) before escalation and high dose (34.1\%) after escalation. Patients in the unchanged group who did not escalate had consistently higher index doses of ICS/LABAs $(P<0.01)$. In addition, the most frequent escalation observed was an ICS dose increase (68.1\%), followed by an add-on of a LABA (27.1\%) and of an LTRM (25.1\%). Only a small fraction of patients switched to a LABA $(0.2 \%)$ or an LTRM $(0.3 \%)$. The percentages of use of other controller medications were very low $(<1 \%)$ in both periods. In the escalation group, fills for rescue medications were common during the pre-escalation (SABA 59.1\%; OCS 29.8\%) and postescalation periods (SABA 56.2\%; OCS 30.7\%). Among those in the unchanged group who did not escalate, $72.1 \%$ were prescribed a SABA during the post-index period. 


\section{TABLE 3 Exacerbation Patterns Before and After} Escalation

\begin{tabular}{|c|c|c|}
\hline \multirow[b]{2}{*}{ Period length, days, mean (SD) } & $\begin{array}{l}\text { Before Escalation } \\
\quad(\mathrm{n}=5,044)\end{array}$ & $\begin{array}{l}\text { After Escalation } \\
\quad(\mathrm{n}=5,044)\end{array}$ \\
\hline & $(105.2)$ & $(138.9)$ \\
\hline $\begin{array}{l}\text { Period length, days, median } \\
\text { (p25, p75) }\end{array}$ & $110 \quad(41,218)$ & $365(101,365)$ \\
\hline $\begin{array}{l}\text { Time to first exacerbation, } \\
\text { days, mean (SD) }\end{array}$ & $(80.8)$ & $(99.2)$ \\
\hline $\begin{array}{l}\text { Time to first exacerbation, } \\
\text { median (p25, p75) }\end{array}$ & $1.00(1.00,66.50)$ & $8.00(1.00,106.0)$ \\
\hline \multicolumn{3}{|c|}{ Patients with asthma exacerbations, $\mathrm{n}(\%)$} \\
\hline Any asthma exacerbation ${ }^{\mathrm{a}}$ & 1,108 & 1,038 \\
\hline With hospitalization & $(2.4)$ & $(2.2)$ \\
\hline $\begin{array}{l}\text { With ED or urgent care facility } \\
\text { visit only }\end{array}$ & $(11.1)$ & $(7.9)$ \\
\hline $\begin{array}{l}\text { With office visit and OCS } \\
\text { pharmacy claim within } 7 \text { days }\end{array}$ & $(86.5)$ & $(89.9)$ \\
\hline
\end{tabular}

Asthma exacerbations PPPY, mean (SD)

\begin{tabular}{l|ll|ll}
\hline Number of asthma exacerbations & 1.60 & $(5.1)$ & 0.75 & $(2.9)$ \\
\hline $\begin{array}{l}\text { Number of exacerbations with } \\
\text { hospitalization }\end{array}$ & 0.03 & $(0.7)$ & 0.03 & $(0.7)$ \\
\hline $\begin{array}{l}\text { Number of exacerbations with } \\
\begin{array}{l}\text { ED or urgent care facility visit } \\
\text { only }\end{array}\end{array}$ & 0.18 & $(1.8)$ & 0.05 & $(0.6)$ \\
\hline $\begin{array}{l}\text { Number of exacerbations with } \\
\text { office visit and OCS pharmacy } \\
\text { claim within 7 days }\end{array}$ & 1.39 & $(4.8)$ & 0.67 & $(2.8)$ \\
\hline Uncontrolled asthma, n (\%) & 2,095 & $(41.5)$ & 2,068 & $(41.0)$ \\
\hline
\end{tabular}

Uncontrolled asthma, ${ }^{\mathrm{b}} \mathrm{n}(\%)$

HCRU per category PPPY, mean (SD)

All-cause

\begin{tabular}{l|rr|rr}
\hline Ambulatory care visits & 24.0 & $(26.7)$ & 19.3 & $(21.3)$ \\
\hline Emergency services visits & 1.3 & $(5.5)$ & 1.0 & $(4.1)$ \\
\hline Inpatient hospital admissions & 0.10 & $(1.09)$ & 0.09 & $(0.85)$ \\
\hline Asthma-related \\
\hline Ambulatory care visits & 8.5 & $(13.4)$ & 4.6 & $(8.1)$ \\
\hline Emergency services visits & 0.4 & $(2.9)$ & 0.1 & $(1.2)$ \\
\hline Inpatient hospital admissions & 0.08 & $(1.04)$ & 0.06 & $(0.80)$ \\
\hline
\end{tabular}

aAsthma exacerbation defined hierarchically as an asthma-related hospitalization, ICD-9-CM code 493.xx in the primary position, an asthma-related ED or urgent care facility visit, ICD-9-CM code 493.xx in the primary position, or an asthmarelated office visit, ICD-9-CM code 493.xx in any position and a pharmacy claim for a systemic (oral or injectable) OCS within \pm 7 days of the claim. Proportions do not account for variation in observation period.

bUncontrolled asthma defined as $\geq 4$ SABA claims per year, $\geq 1$ claim for an OCS, or $\geq 1$ asthma-related ED or hospitalization with ICD-9-CM code 493.xx in the primary position.

CUtilization reported PPPY to account for variable observation time among all patients.

$E D=$ emergency department; HCRU = health care resource utilization; ICD-9-CM= International Classification of Diseases, Ninth Revision, Clinical Modification; ICS = inhaled corticosteroids; OCS = oral cortacosteroid; $P P P Y=$ per patient per year; $S A B A=$ short-acting beta-2 agonist; $S D=$ standard deviation.

\section{Outcomes}

Exacerbations and Uncontrolled Asthma. During the mean (SD) pre-escalation period of 136.1 (105.2) days, $22.0 \%$ of patients who escalated treatment had at least 1 exacerbation, with the majority of exacerbations (86.5\%) identified by an office visit with an OCS pharmacy claim within 7 days (Table 3). During the mean postescalation period of 251.6 (138.9) days, $20.6 \%$ of patients had an exacerbation, with most (89.9\%) identified by an office visit with an OCS claim. In addition, $41.5 \%$ of patients experienced uncontrolled asthma before escalation and $41.0 \%$ after escalation.

Health Care Resource Utilization. Use of ambulatory care services was common in the pre- and postescalation periods (Table 3). Before escalation, the all-cause and asthma-related number of ambulatory visits were 24.0 (26.7) and 8.5 (13.4) PPPY, respectively. After escalation, the all-cause and asthmarelated ambulatory care visits were 19.3 (21.3) and 4.6 (8.1) PPPY, respectively. Emergency services use was lower in the postescalation period (1.0 and 0.1 visits PPPY for all-cause and asthma-related visits) compared with the pre-escalation period (1.3 and 0.4 visits PPPY for all-cause and asthma-related visits, respectively). Inpatient admissions were infrequent, at 0.1 PPPY, during each period for all-cause and asthma-related admissions. Cost data are presented in Appendix B.

\section{Discussion}

In this study, asthma treatment patterns and outcomes were examined in a real-world setting. Pre- and postescalation treatment patterns and outcomes, including exacerbation events, health care utilization, and costs, were examined. This study contributes to the literature by providing an in-depth description of outcomes after escalation of index ICS-containing treatment, a rarely examined perspective. These data provide a foundation for future research regarding improvements in asthma management.

Treatment escalations included in this analysis were defined by step therapy guidelines for asthma control. ${ }^{5}$ Among patients meeting initial inclusion criteria for this study, $14.4 \%$ had evidence of treatment escalation; $62.4 \%$ had no change in treatment regimen; and $23.1 \%$ had a step-down in treatment within 1 year of initiation of an ICS-containing therapy. The most common types of regimen escalations were an ICS dose increase, an LABA add-on, or an LTRM add-on. These findings are similar to results reported previously in a sample of patients with asthma. ${ }^{22}$ The majority of the asthma population identified for this study did not escalate their index therapies within 1 year, yet a large proportion of patients (37.5\%) did have some form of dose adjustment (escalation or step-down) per the current NHLBI treatment guidelines. ${ }^{5}$ The index dosages observed for ICS were similar among the escalated versus unchanged group; however, higher index dosages of ICS/ LABAs were observed among those who did not require escalation. Although the primary study cohort of interest, those who escalated therapy, did not represent a majority of the total sample, the results demonstrate that gaps in care remain despite escalation. 
A substantial concern in asthma management is exacerbation, which can still occur for some patients after treatment escalation. We found that approximately 1 in $5(21 \%)$ patients experienced at least 1 exacerbation during the mean (SD) postescalation period of 251.6 (138.9) days, and the overall exacerbation rate among the escalation cohort after escalation was 0.75 PPPY. Despite minor differences in definitions of treatment escalation and exacerbation, these findings are similar to results from 2 previous studies, the first reporting that approximately $16 \%$ of patients experienced at least 1 exacerbation after initiating a step-up in therapy, ${ }^{17}$ and a second that reported an exacerbation rate of 0.67 PPPY among patients who were treated with "high-intensity" therapy. ${ }^{23}$ Findings from this study similarly demonstrate suboptimal treatment outcomes for a sizable group of patients despite an escalation in treatment.

The central aim of asthma management is achieving and maintaining control of asthma symptoms. ${ }^{5,6}$ Although our study cohort was not limited to patients experiencing persistent asthma, our estimate of the prevalence of uncontrolled asthma was similar to that from a study by Zeiger et al. (2016), which reported that $42.6 \%$ of patients with persistent asthma were uncontrolled. ${ }^{24}$ Based on the evidence of fills for OCS or SABAs, or ED or inpatient utilization, $41.5 \%$ of patients in this study had uncontrolled asthma during the pre-escalation period (mean 136.1 [105.2] days), and during the postescalation period (mean 251.6 [138.9] days), 41.0\% had uncontrolled asthma. Thus, a high percentage of patients may continue to have uncontrolled asthma even with escalated treatment, indicating a gap in current asthma management.

Zeiger et al. also provided recent data regarding health care resource utilization and costs associated with patients with asthma newly initiating ICS treatment, before and after treatment escalation (Appendix B). ${ }^{24}$ Ambulatory care was common in the pre- and postescalation periods. In the pre-escalation period, the PPPY all-cause and asthma-related number of ambulatory visits was 24.0 (26.7) and 8.5 (13.4), respectively. Utilization was lower in the postescalation period with PPPY all-cause and asthma-related ambulatory care visits of 19.3 (21.3) and 4.6 (8.1), respectively. Sullivan et al. (2015) demonstrated similar rates of ambulatory care utilization, with 16-34 visits per year, although in contrast to our study, utilization rates reported by Sullivan et al. increased as treatment intensity increased. ${ }^{16}$ Sullivan et al. proposed that despite a reduction in exacerbation events, as was also observed in our study, the treatments used by most patients were suboptimal, and patients consistently sought additional treatment options, primarily through ambulatory care visits. ${ }^{16}$ Emergency and inpatient care were infrequent in our study, at less than 1.5 visits PPPY, a rate also similar to previous reports. ${ }^{16}$
The patients included in our study were relatively young and healthy, with low comorbidity scores and a low rate of inpatient stays (0.1 PPPY) for all-cause and asthma-related care. An asthma diagnosis, in any position, on the inpatient claim was required to be considered an asthma-related inpatient stay. However, it is possible that inpatient care related to other conditions was incorrectly attributed to asthma. It is also possible that patients were prescribed ICS medications for other nonasthma respiratory conditions, such as respiratory infections (Appendix A), which were prevalent in the entire sample and slightly more common among those who escalated treatment.

Over recent years, studies using several different databases have provided consistent evidence that care for patients with persistent uncontrolled asthma imposes substantial burden to the health care system. ${ }^{11,13,16}$ As newer therapies become available, further research may be warranted to understand the value of these agents in mitigating the gap in asthma-related outcomes and reducing this burden.

\section{Limitations}

This study has some limitiations to consider. Administrative claims are submitted for the purpose of payment rather than research and have certain limitations, including potential coding errors and incomplete data. For example, pharmacotherapies are not captured if they were obtained outside of the insurance system (e.g., out-of-pocket payment for generic medications or medications obtained as free samples from physicians). The identification of exacerbations based on resource use and systemic corticosteroid use may over- or underestimate the true exacerbation rate due to the lack of clinical verification. The definitions for asthma control involved proxies defined by claims data and may not be consistent with definitions used in clinical practice.

It is also important to note that clinical information such as lung function test results and patient-reported symptoms such as wheezing, cough, shortness of breath, and nighttime awakening could not be captured in the database. Similarly, asthma medications may have been prescribed for other nonasthma respiratory conditions observed in this population. In addition, as there was no minimum requirement for the postescalation period, patients with longer postescalation observation might be more likely to meet the criteria we used to define uncontrolled asthma.

The study population was selected from enrollees in commercial health insurance plans, so results may not be generalizable to noncommercially insured patients (i.e., Medicare, Medicaid, or uninsured) or patients outside the database. 
Finally, the analyses were descriptive in nature, and statistical testing was not performed for outcome measures. Thus, restraint should be used in drawing conclusions. Furthermore, non-PPPY outcomes should be considered with the differing length of respective observation periods in mind. The PPPY exacerbation rate and HCRU appear to be higher in the pre-escalation period relative to the postescalation period; however, additional research with statistical testing and multivariable analysis is needed to further evaluate the association of treatment escalation with outcomes and to investigate the effect of other factors such as treatment adherence on patient outcomes.

\section{Conclusions}

One in 7 asthma patients initiating treatment on an ICS or ICS-containing regimen had treatment escalation within a year following the index therapy. One in 5 of those patients who escalated treatment had an exacerbation, and 2 in 5 had uncontrolled asthma after treatment escalation. Although treatment escalation does not equate to treatment failure, the continuing burden of exacerbations and evidence of uncontrolled asthma after escalation suggest a remaining unmet need among asthma patients who escalate their ICS-containing treatments and remain uncontrolled.

\section{Authors}

LINDSAY G. S. BENGTSON, PhD; FELIX CAO, PhD; ERIN M. HULBERT, MS; RYAN WOLBECK, MS; CAITLIN A. ELLIOTT, MS; and AMI R. BUIKEMA, MPH, Optum, Eden Prairie, Minnesota. YANNI YU, DSc, and WEIJIA WANG, MSc, Boehringer-Ingelheim, Ridgefield, Connecticut.

AUTHOR CORRESPONDENCE: Lindsay G. S. Bengtson, PhD, Optum, 11000 Optum Cir., Eden Prairie, MN 55344.

Tel.: 952.205.7717; E-mail: lindsay.bengtson@optum.com.

\section{DISCLOSURES}

This study was sponsored and funded by Boehringer-Ingelheim, which contracted with Optum to conduct the research. The sponsor collaborated with Optum on the preparation, writing, revision, and approval of the manuscript. Bengston, Cao, Hulbert, Wolbeck, Elliott, and Buikema are employees of Optum. Yu and Wang are employed by Boehringer-Ingelheim.

Study concept and design were contributed by Bengston, Yu, and Wang. Cao, Hulbert, and Wolbeck collected the data, and data analysis was performed by Bengston, Yu, and Wang. The manuscript was written by Bengston, along with $\mathrm{Yu}$ and Wang, and revised by Bengston, Yu, and Wang, along with the other authors.

\section{ACKNOWLEDGMENTS}

Medical writing services were provided by Caroline Jennermann, MS, an employee of Optum, as contracted by Boehringer Ingelheim.

\section{REFERENCES}

1. Global Asthma Network. The Global Asthma Report 2014. Auckland, New Zealand: Global Asthma Network; 2014. Available at: http://www.globalasthmareport.org/resources/Global_Asthma_Report_2014.pdf. Accessed September 20, 2017.

2. Centers for Disease Control and Prevention. Most recent asthma data. Prevalence. National current asthma prevalence (2015). Page updated June 2017. Available at: https://www.cdc.gov/asthma/most_recent_data.htm. Accessed September 20, 2017.

3. Centers for Disease Control and Prevention. Most recent asthma data. Health care use. National health care use. Page updated June 2017. Available at: https://www.cdc.gov/asthma/most_recent_data.htm. Accessed September 20, 2017.

4. Barnett SB, Nurmagambetov TA. Costs of asthma in the United States: 2002-2007. J Allergy Clin Immunol. 2011;127(1):145-52.

5. National Heart, Lung, and Blood Institute, National Asthma Education and Prevention Program. Expert panel report 3. Guidelines for the diagnosis and management of asthma. Full report 2007. August 28, 2007. Available at: https://www.nhlbi.nih.gov/files/docs/guidelines/asthgdln.pdf. Accessed September 20, 2017

6. Global Initiative for Asthma. Global strategy for asthma management and prevention. 2016 update. Available at: http://ginasthma.org/wp-content/ uploads/2016/04/GINA-2016-main-report_tracked.pdf. Accessed

September 20, 2017.

7. Chauhan BF, Chartrand C, Ni Chroinin M, Milan SJ, Ducharme FM. Addition of long-acting beta2-agonists to inhaled corticosteroids for chronic asthma in children. Cochrane Database System Rev. 2015;11:CD007949.

8. Chung KF, Wenzel SE, Brozek JL, et al. International ERS/ATS guidelines on definition, evaluation and treatment of severe asthma. Eur Respir J. 2014:43(2):343-73.

9. Fuhlbrigge A, Reed ML, Stempel DA, et al. The status of asthma control in the U.S. adult population. Allergy Asthma Proc. 2009;30(5):529-33.

10. Calhoun WJ, Haselkorn T, Mink DR, Miller DP, Dorenbaum A, Zeiger RS. Clinical burden and predictors of asthma exacerbations in patients on guideline-based steps 4-6 asthma therapy in the TENOR cohort. J Allergy Clin Immunol Pract. 2014;2(2):193-200.

11. Ivanova JI, Bergman R, Birnbaum HG, Colice GL, Silverman RA, McLaurin K. Effect of asthma exacerbations on health care costs among asthmatic patients with moderate and severe persistent asthma. J Allergy Clin Immunol. 2012;129(5):1229-35.

12. Custovic A, Johnston SL, Pavord I, et al. EAACI position statement on asthma exacerbations and severe asthma. Allergy. 2013;68(12):1520-31.

13. Chastek B, Korrer S, Nagar SP, et al. Economic burden of illness among patients with severe asthma in a managed care setting. J Manag Care Spec Pharm. 2016;22(7):848-61. Available at: http://www.jmcp.org/doi/10.18553/ jmcp.2016.22.7.848.

14. Suruki RY, Boudiaf N, Ortega HG. Retrospective cohort analysis of healthcare claims in the United States characterizing asthma exacerbations in paediatric patients. World Allergy Organ J. 2016;9:18.

15. Sullivan PW, Slejko JF, Ghushchyan VH, et al. The relationship between asthma, asthma control and economic outcomes in the United States. J Asthma. 2014;51(7):769-78.

16. Sullivan PW, Campbell JD, Ghushchyan VH, Globe G. Outcomes before and after treatment escalation to Global Initiative for Asthma steps 4 and 5 in severe asthma. Ann Allergy Asthma Immunol. 2015;114(6):462-69.

17. Israel E, Roche N, Martin RJ, et al. Increased dose of inhaled corticosteroid versus add-on long-acting beta-agonist for step-up therapy is asthma. Ann Am Thorac Soc. 2015;12(6):798-806.

18. U.S. Census Bureau. Census regions and divisions of the United States. Available at: http://www2.census.gov/geo/pdfs/maps-data/maps/reference/ us_regdiv.pdf. Accessed September 20, 2017. 
19. Quan H, Li B, Couris CM, et al. Updating and validating the Charlson comorbidity index and score for risk adjustment in hospital discharge abstracts using data from 6 countries. Am J Epidemiol. 2011;73(6):676-82.

20. Marceau C, Lemiere C, Berbiche D, Perrault S, Blais L. Persistence, adherence, and effectiveness of combination therapy among adult patients with asthma. J Allergy Clin Immunol. 2006;118(3):574-81.

21. Stempel DA, McLaughin TP, Stanford RH, Fuhlbrigge AL. Patterns of asthma control: a 3-year analysis of patient claims. J Allergy Clin Immunol. 2005; 115(5):935-39.

22. Tan H, Sarawate C, Singer J, et al. Impact of asthma controller medications on clinical, economic, and patient-reported outcomes. Mayo Clin Proc. 2009;84(8):675-84.
23. Schatz M, Zeiger RS, Vollmer WM, et al. The controller-to-total asthma medication ratio is associated with patient-centered as well as utilization outcomes. Chest. 2006;130(1):43-50

24. Zeiger RS, Schatz M, Dalal AA, et al. Utilization and costs of severe uncontrolled asthma in a managed-care setting. J Allergy Clin Immunol. 2016;4(1):120-29

25. Foote ER, Singleton RJ, Holman RC, et al. Lower respiratory tract infection hospitalizations among American Indian/Alaska Native children and the general United States child population. Int J Circumpolar Health. 2015;74:29256

26. U.S. Bureau of Labor Statistics. Consumer Price Index for all urban consumers (current series). U.S. medical care services. 2014. Available at: https://data.bls.gov/cgi-bin/surveymost?cu. Accessed October 3, 2017. 


\section{APPENDIX A ICD-9-CM Codes}

Claims identifying chronic obstructive pulmonary disease (ICD-9-CM 491.xx, 492.x, 496, 506.4, 518.1, and 518.2); cystic fibrosis (ICD-9-CM 277.0x); and interstitial lung disease (ICD-9-CM 135, 162, 237.7, 272.7, 277.3, 277.8, 416.0, 446.21, 446.4, 495, 500-505, 506.4, 508.1, 508.8, 516, 519.9, 517.2, 517.8, 518.3, and 555).

Codes identifying lower respiratory tract infection: pulmonary tuberculosis (011); pulmonary anthrax (022.1); and pulmonary diseases caused by Mycobacterium (031.0), whooping cough (033), respiratory syncytial virus (079.6), syphilis of lung (095.1), acute bronchitis (466.0), acute bronchiolitis (466.1), pneumonia (480-486), influenza (487), influenza due to identified 2009 H1Nl influenza virus (488.1), emphysema (510), pleurisy with effusion (511.1), abscess of lung and mediastinum (513), rheumatic pneumonia (517.1), or congenital pneumonia (770.0) during the pre-ICS baseline period. ${ }^{25}$ ICD-9-CM = International Classification of Diseases, Ninth Revision, Clinical Modification.

\section{APPENDIX B Supplementary Methods and Results: Costs Analyses}

Costs were computed as the combined health plan and patient paid amounts on a per-patient-per-year (PPPY) basis in the pre- and postescalation periods and include medical costs, pharmacy costs, and total costs (medical and pharmacy costs combined). Medical costs were further categorized as ambulatory costs, emergency department costs, inpatient costs, and other costs. Costs were adjusted to 2014 U.S. dollars using the annual medical care component of the Consumer Price Index to reflect inflation between 2009 and 2014.26

Mean all-cause health care costs were $\$ 12,904$ PPPY during the pre-escalation period, with all-cause ambulatory care costs representing $51.9 \%$ of the total. During the postescalation period, the all-cause costs were $\$ 11,315$ PPPY, with ambulatory care costs representing nearly half (48.3\%) of the total.

\begin{tabular}{|c|c|c|c|c|}
\hline \multicolumn{5}{|c|}{ Health Care Costs, Escalation Group ${ }^{a}$} \\
\hline & \multicolumn{2}{|c|}{$\begin{array}{c}\text { Before Escalation } \\
(\mathrm{n}=5,044, \$)\end{array}$} & \multicolumn{2}{|c|}{$\begin{array}{l}\text { After Escalation } \\
(\mathrm{n}=5,044, \$)\end{array}$} \\
\hline \multicolumn{5}{|l|}{ All-cause cost PPPY, mean (SD) } \\
\hline Total health care costs & 12,904 & $(42,011)$ & 11,315 & $(29,189)$ \\
\hline Pharmacy costs & 3,713 & $(5,045)$ & 3,589 & $(6,027)$ \\
\hline Medical costs & 9,191 & $(40,891)$ & 7,726 & $(27,846)$ \\
\hline Ambulatory costs $\mathrm{s}^{\mathrm{b}}$ & 6,706 & $(23,128)$ & 5,463 & $(12,581)$ \\
\hline Emergency services costs & 270 & $(1,505)$ & 192 & $(1,325)$ \\
\hline Inpatient costs & 1,762 & $(27,040)$ & 1,607 & $(22,377)$ \\
\hline Other medical costs & 452 & $(2,445)$ & 464 & $(3,763)$ \\
\hline \multicolumn{5}{|l|}{ Asthma-related costw PPPY, mean (SD) } \\
\hline Total asthma-related health care costs & $\begin{array}{r}6,119 \\
47.4 \% \text { of tot }\end{array}$ & $\begin{array}{l}(34,559) \\
1 \text {-cause costs }\end{array}$ & $\begin{array}{r}4,413 \\
39.0 \% \text { of tot }\end{array}$ & $\begin{array}{l}(22,589) \\
1 \text {-cause costs }\end{array}$ \\
\hline Asthma-related pharmacy costs & 2,018 & $(2,395)$ & 1,823 & $(2,195)$ \\
\hline Asthma-related medical costs & 4,100 & $(34,302)$ & 2,591 & $(22,354)$ \\
\hline Ambulatory costs & 2,485 & $(17,182)$ & 1,369 & $(4,930)$ \\
\hline Emergency services costs & 92 & $(1,003)$ & 30 & $(766)$ \\
\hline Inpatient costs & 1,463 & $(25,646)$ & 1,137 & $(21,666)$ \\
\hline Other medical costs & 60 & $(554)$ & 55 & $(1,183)$ \\
\hline \multicolumn{5}{|c|}{$\begin{array}{l}\text { Note: Health care costs are combined health plan and patient paid amounts. } \\
\text { aCosts were adjusted to } 2014 \text { U.S. dollars using the annual medical care component of the Consumer Price Index to reflect inflation between the earliest (2009) and la } \\
\text { (2014) year of data. } \\
\text { bIncludes physician office and outpatient visits. } \\
\text { PPPY=per patient per year; SD = standard deviation. }\end{array}$} \\
\hline
\end{tabular}

